\title{
Innate Preference for Host-Odor Blends Modulates Degree of Anthropophagy of Anopheles gambiae sensu lato (Diptera: Culicidae)
}

\author{
TEUN DEKKER, ${ }^{1}$ WILLEM TAKKEN, AND MARIETA A. H. BRAKS ${ }^{2}$
}

Laboratory of Entomology, Wageningen University, P.O. Box 8031, 6700 EH Wageningen, The Netherlands

J. Med. Entomol. 38(6): 868-871 (2001)

\begin{abstract}
In field studies, Anopheles gambiae Giles sensu stricto obtains most blood meals from humans, whereas Anopheles quadriannulatus Theobald bites predominantly bovids. We investigated whether host odors modulate the host preference of these mosquito species. In a dual-choice olfactometer, mosquitoes were given a choice between clean air and putative host-specific odor blends. An. gambiae chose 'human odor' over clean air and clean air over 'cow odor.' Although Anopheles quadriannulatus did not choose cow odor over clean air, it chose clean air over human odor. Cheese odor, which attracted An. gambiae, did not result in higher trap catch of An. quadriannulatus. We conclude that the degree of anthropophagy of An. gambiae s.l. has an innate olfactory basis.
\end{abstract}

KEY WORDS Anopheles gambiae, Anopheles quadriannulatus, behavior, host choice, odors

THE HUMAN-BITING INDEX, which is the proportion of blood meals taken from a human host, has a large impact on a mosquito species' vectorial capacity for malaria. This is illustrated by the fact that the world's major malaria vectors all feed predominantly on humans (Garrett-Jones 1964). Species within the Anophese gambiae Giles complex differ highly in their vectorial capacity for malaria. Because the sibling species appear equally susceptible to Plasmodium falciparum Welch infection (Takken et al. 1999), differences in the human biting index of the sibling species mostly determine their status as malaria vectors. Anopheles gambiae sensu stricto (henceforth termed An. gambiae) is the most important vector of malaria in Africa and is highly anthropophagic, whereas its nonvector sibling, Anopheles quadriannulatus Theobald, feeds for $>95 \%$ on bovids (White 1974, Sharp et al. 1984, Gillies and Coetzee 1987). Although odors are evidently used in host finding by An. gambiae s.l. (Takken and Knols 1999), their role in host choice has been disputed (e.g., Diatta et al. 1998). We investigated whether host choice by An. gambiae and An. quadriannulatus is modulated by an innate preference for host-odor blends from their 'preferred' hosts.

\section{Materials and Methods}

Mosquitoes. An. gambiae originating from Suakoko, Liberia was established in the laboratory in 1987 (courtesy of M. Coluzzi, Rome, Italy). An. quadriannulatus originating from Skukuza, South Africa (SKU-

\footnotetext{
${ }^{1}$ Current addresses: Department of Entomology, University of California, Riverside, CA 92521 (e-mail: tdekker@ucracl.ucr.edu).

${ }^{2}$ Florida Medical Entomology Research Laboratory, 200 9th Street SE, Vero Beach, FL 32962.
}

QUA) was tested in the 12-18th generation after introduction in the laboratory. Mosquitoes were reared at $80 \% \mathrm{RH}$ and a photoperiod of 12:12 (L:D) h without artificial dusk period. Larvae were reared on Baby Fish Food (Tetramin, Melle, Germany). Adults were kept in 30 by 30 by $30-\mathrm{cm}$ gauze cages (An. gambiae) or 50 $\mathrm{cm}$ high by $30-\mathrm{cm}$ diameter buckets with a mosquito gauze lid (An. quadriannulatus) and provided with a $6 \%$ glucose solution. Females of both species were blood fed on human arm only.

Bioassay and Procedures. We tested unfed, mated female mosquitoes of 5-12 d old, which had not had prior exposure to odors in a bioassay setup. Mosquitoes were transferred to release cages $15 \mathrm{~h}$ before testing. Each release cage contained 50 mosquitoes, 25 of each species. An. gambiae was dusted with a green fluorescent pigment (Day-Glo Color Co., Cleveland, $\mathrm{OH}$ ) to be able to distinguish it from the morphologically identical An. quadriannulatus. A water-soaked piece of cotton was placed on top of the cage to prevent desiccation. We used a dual-choice olfactometer comprised of a transparant Lexan flight chamber of 160 long by 60 wide by $60 \mathrm{~cm}$ high (Braks and Takken 1999). Mosquitoes released at one end of the flight chamber had a choice of two airstreams of $20 \mathrm{~cm} / \mathrm{s}$ emanating from two ports at the opposite end. Flying upwind in these ports resulted in capture in a 'no-exit' device. After $25 \mathrm{~min}$, mosquitoes remaining in the flight chamber were removed. The number of each species in treatment trap (T), control trap (C) and release cage (nonresponders) were counted. The release cage and trapping devices were replaced with clean ones, and a new test could start.

Odors. We tested $\mathrm{CO}_{2}$ at $4.5 \%$, the concentration expired by humans and cows, and blends of 'human odor,' 'cow odor,' and cheese odor. Clean air and $\mathrm{CO}_{2}$ 


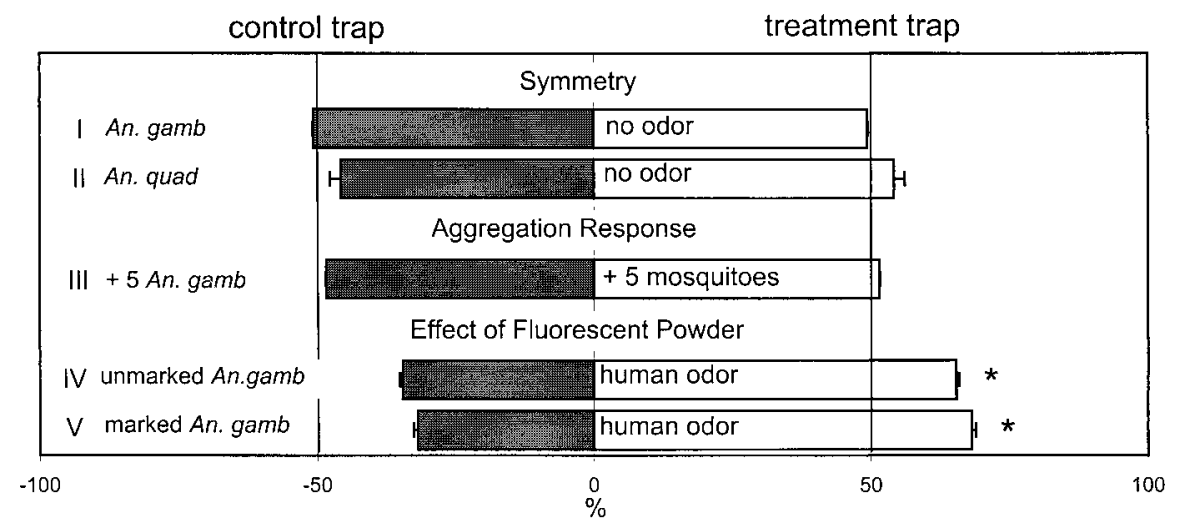

Fig. 1. Distribution of catches over control and treatment trap. Each bar represents 100\%. I and II - trap catches of An. gambiae (An. gamb) and An. quadriannulatus (An. quad) with both traps unbaited. III - trap catch of An. gambiae with five mosquitoes in the treatment trap. IV and V -trap catch of unmarked (IV) and fluorescent-powder marked (V) An. gambiae with one trap baited with human odor the other unbaited. *, $P<0.05$.

(medical air and $100 \% \mathrm{CO}_{2}$, respectively, Hoek-Loos, The Netherlands) were mixed in a 100 -liter Teflon gas-sampling bag (Tedlar). We added $10 \mathrm{ml}$ of distilled water to achieve a near saturation moisture level. Air from the gasbag was pumped through Teflon tubing (5 mm wide) via a flow meter (Sho-rate, Brooks Instrumental B.V., The Netherlands), into the traps at 230 $\mathrm{ml} / \mathrm{min}$. To obtain a blend of human odor a male Caucasian (28 yr old) wore a nylon sock on his foot for eight consecutive hours. The socks were stored in an airtight glass jar at room temperature for $12 \mathrm{~h}$ before testing. To obtain a cow-odor blend a nylon sock was wrapped around the upper hind leg of a cow. After $12 \mathrm{~h}$ the sock was removed and stored for $2 \mathrm{~h}$ in an airtight glass jar. Socks were put in the trap directly before the experiments and used singly. We used extracts of Hüttenberger Mainzer Cheese ( $100 \mathrm{~g}$ in 1 liter ethyl alcohol, courtesy Bayer AG, Leverkussen). One hundred microliters of the extract was applied on a sandblasted glass slide of 2 by $5 \mathrm{~cm}$. When the alcohol was evaporated, the slide was inserted into the no-exit device and tested against clean air.

Treatments and Analysis. Baited traps were tested against clean-air controls. Control and treatment sides were alternated between experiments. Each treatment was repeated eight times. In a separate series we tested the symmetry of the setup and whether fluorescent dust on An. gambiae affected choice between human odor and clean air. We also tested for a possible aggregative response by scoring the choice of $A n$. gambiae between two traps, one of which contained five females.

Two parameters were used to evaluate the results of the tests: the proportion of each species in the test trap calculated as $T /(T+C)$, and the total proportion caught, calculated as $(T+C) /(25$ - nonresponders $)$. Data were arcsine transformed and differences between treatment and control were analyzed with a one-sample $t$-test (two-tailed) with $\mu=0.5$ and $\alpha=$ 0.05 .

\section{Results and Discussion}

Figure 1 shows that the olfactometer is symmetric and that mosquitoes, which were already present in one of the traps at the start of the experiment, did not affect symmetry. It also shows that using fluorescent powder on An. gambiae did not change their response to human odor, which enabled simultaneous testing of An. gambiae and the morphologically identical An. quadriannulatus.

The total proportion An. quadriannulatus that entered the traps was at most 0.28 , whereas irrespective of treatment this proportion was around 0.70 for $A n$. gambiae. We observed An. quadriannulatus hovering in front of the trap opening, without entering. Other endophagic species tested, including Aedes aegypti (L.) (Dekker et al. 2001) and Culex quinquefasciatus Say (T.D. and W.T., unpublished data), quickly entered baited traps. Probably lack of innate endophagy impaired trap entry by An. quadriannulatus in response to host odor.

Trap catches with host odors partially reflected host choice in the field (Fig. 2). An. gambiae chose human odor over clean air $(P=0.02)$, whereas An. quadriannulatus chose clean air over human odor $(P=$ 0.002). 'Cow odor,' however, caught fewer An. gambiae than the clean-air control $(P=0.005)$. Unexpectedly, An. quadriannulatus did not choose cow odor over clean air, which was possibly caused by incompleteness of the cow odor blend. $\mathrm{CO}_{2}$, an important host stimulus for An. quadriannulatus (Dekker and Takken 1998), may be necessary to increase trap catch of An. quadriannulatus with cow odor in a similar fashion as $\mathrm{CO}_{2}$ increases trap catches with 1-octen3-ol for several mosquito species (Takken and Kline 1989). A recent study with cow odor and $\mathrm{CO}_{2}$ supports this hypothesis (H. V. Pates, Wageningen University, the Netherlands, personal communication).

The reduced trap catch of An. gambiae and An. quadriannulatus with cow odor and human odor, respectively, seems to suggest that odor of "nonpre- 


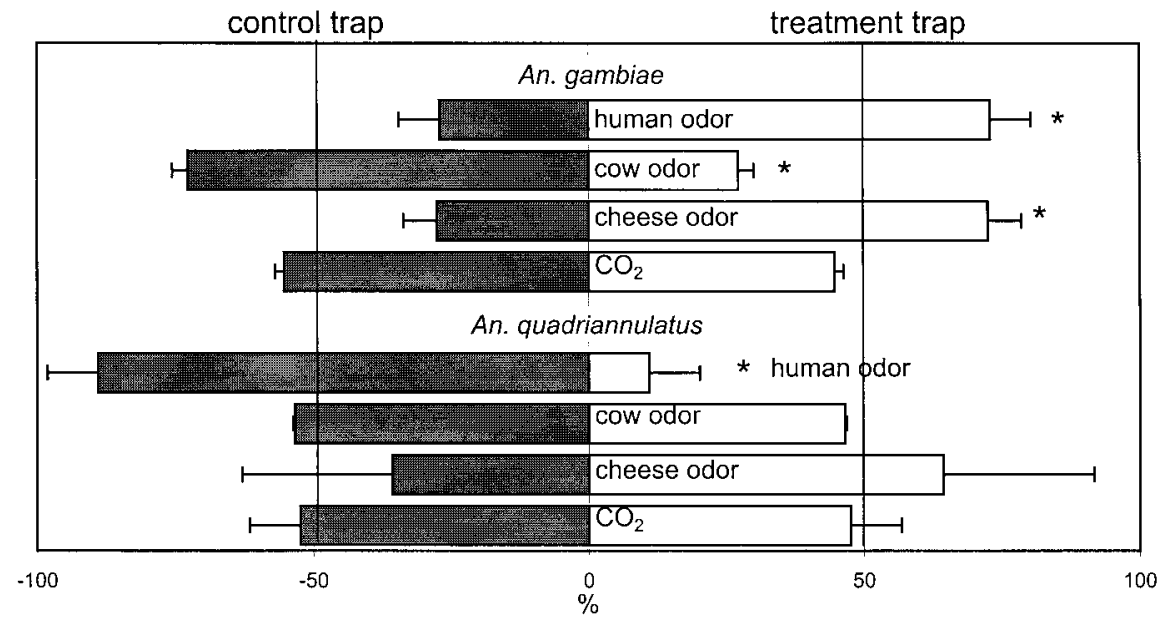

Fig. 2. Distribution of the trap catches of An. gambiae and An. quadriannulatus with odors in the treatment trap and no odor in the control trap. Each bar represents $100 \%$. *, $P<0.05$.

ferred' hosts are repellent to these species. Vale (1979) found that nonhost (human) odor repel tsetse, Glossina morsitans morsitans Westwood. Similarly, field data indicate that nonhost odor repel An. gambiae (Costantini et al. 1998) and An. quadriannulatus (Dekker and Takken 1998). In our setup, however, choice could also have been influenced by exposure of the mosquitoes to the experimenter's odor at release, or by encounters of odor filaments from previous tests still present in the flight chamber. However, although cow odor clearly contained ammonia, an attractant for $A n$. gambiae at a wide range of concentrations (Braks et al. 2001), trap catches of An. gambiae were lower with cow odor than with clean air. It is concluded that some factor in the cow odor blend reduced the attractiveness of ammonia and possibly other attractants to $A n$. gambiae.

Cheese odor, previously shown to attract An. gambiae (Knols and De Jong 1996), was not attractive for An. quadriannulatus $(P=0.08$ versus $P=0.01$ for $A n$. gambiae; Fig. 2). This was likely caused by the high standard deviation due to the low trap-entry rates of An. quadriannulatus. Otherwise, the results could imply that the cheese-odor blend resembles human odor more than cow odor.

Carbon dioxide did not result in higher trap catch than the control, despite the fact that $\mathrm{CO}_{2}$ induces trap catches of seemingly all mosquito species in the field (Mboera and Takken 1997). Field studies also have shown a differential attraction of species of the $A n$. gambiae complex to $\mathrm{CO}_{2}$ (e.g., Snow 1970, Costantini et al. 1996, Dekker and Takken 1998). This corroborates findings of Dekker et al. (2001). They used electric nets placed in front of the trap entry to demonstrate that An. gambiae s.s. was attracted to $\mathrm{CO}_{2}$, but did not enter $\mathrm{CO}_{2}$-baited traps at a higher rate than the control trap. We conclude that our setup is not well suited for testing responses of mosquitoes to $\mathrm{CO}_{2}$ and recommend further studies on the orientation behavior of mosquitoes to odor plumes of different constituents.

One might argue that feeding of our An. quadriannulatus strain on human arm may have caused a shift to more anthropophagic behavior, similar to the change in host preference of An. atroparvus from human to rabbit (Laarman 1958). Three factors, however, indicate that such a selection had not occurred in our An. quadriannulatus strain. First, blood feeding of An. gambiae and An. quadriannulatus for colony maintenance showed a marked contrast between both species. An. gambiae engorged within 3 min, whereas blood feeding of An. quadriannulatus on human arm took up to 30 min and only by frequently breathing into the cage were females induced to bite. Second, our results show that An. quadriannulatus and $A n$. gambiae responded very differently to host odors. Finally, Pates et al. (2001) compared host-odor choice of a membrane-fed strain An. quadriannulatus with our human-fed strain and found no differences between the two strains.

Factors that influence the human biting index of a mosquito species include the relative availability of host species (Garrett-Jones et al. 1980, and references therein) and the innate degree of endophily (Trpis and Hauserman 1978, Coluzzi et al. 1979, Diatta et al. 1998). Our results strongly suggest that innate preferences for putative host-specific odor blends also modulate the degree of anthropophagy of An. gambiae s.l. Further studies on role of olfaction in host choice by mosquitoes may prove valuable for development of sampling methods that specifically lure anthropophilic species.

\section{Acknowledgments}

We thank Leo Koopman, Frans van Aggelen, and André Gidding for rearing An. gambiae, and Helen Pates for allowing us to mention some unpublished data. We acknowledge 
Miriam Cooperband for critically reading the manuscript. The experimental farm, The Ossekampen, of Wageningen University is gracefully acknowledged for having provided the cow odor. This study was supported by the Praeventiefonds, the Netherlands and Bayer AG, Leverkusen, Germany.

\section{References Cited}

Braks, M.A.H., and W. Takken. 1999. Incubated human sweat but not fresh sweat attracts the malaria mosquito Anopheles gambiae sensu stricto. J. Chem. Ecol. 25: 663672.

Braks, M.A.H., J. Meijerink, and W. Takken. 2001. The role of human sweat components, ammonia and L-lactic acid, in the behaviour of the anthropophilic malaria mosquito, Anopheles gambiae (Culicidae; Diptera). Physiol. Entomol. 26: 142-148.

Coluzzi, M., A. Sabatini, V. Petrarca, and M. A. Di Deco. 1979. Chromosomal differentiation and adaptation to human environments in the Anopheles gambiae complex. Trans. R. Soc. Trop. Med. Hyg. 73: 483- 497.

Costantini, C., G. Gibson, N. Sagnon, A. Della Torre, J. Brady, and M. Coluzzi. 1996. Mosquito responses to carbon dioxide in a West African Sudan savanna village. Med. Vet. Entomol. 10: 220-227.

Costantini, C., N. Sagnon, A. Della Torre, M. Diallo, and J. Brady. 1998. Odor-mediated host preferences of West African mosquitoes, with particular reference to malaria vectors. Am. J. Trop. Med. Hyg. 58: 56-63.

Dekker, T., and W. Takken. 1998. Differential responses of mosquito sibling species Anopheles arabiensis and An. quadriannulatus to carbon dioxide, a man or a calf. Med. Vet. Entomol. 12: 136-140.

Dekker, T., W. Takken, and R. T. Cardé. 2001. Structure of host-odour plumes influences catch of Anopheles gambiae s.s. and Aedes aegypti in a dual-choice olfactometer. Physiol. Entomol. 26: 124-134.

Diatta, M., A. Spiegel, L. Lochouarn, and D. Fontenille. 1998. Similar feeding preferences of Anopheles gambiae and A. arabiensis in Senegal. Trans. R. Soc. Trop. Med. Hyg. 92: 270-272.

Garrett-Jones, C. 1964. The human blood index of malaria vectors in relation to epidemiological assessment. Bull. W.H.O. 30: 241-261.

Garret-Jones, C., P.F.L. Boreham, and C. P. Pant. 1980. Feeding habits of anophelines (Diptera: Culicidae) in 1971-78, with reference to the human blood index: a review. Bull. Entomol. Res. 70: 165-185.
Gillies, M. T., and M. Coetzee. 1987. Supplement to the Anophelinae of Africa south of the Sahara (Afrotropical Region). Publication of the South African Institute of Medical Res., Johannesburg, no. 55.

Knols, B.G.J., and R. De Jong. 1996. Limburger cheese as an attractant for the malaria mosquito Anopheles gambiae s.s. Paras. Today 12: 159-161.

Laarman, J. J. 1958. The host-seeking behaviour of anopheline mosquityoes. Trop. Geog. Med. 10: 293-305.

Mboera, L.E.G., and W. Takken. 1997. Carbon dioxide chemotropism in mosquitoes (Diptera: Culicidae) and its potential in vector surveillance and management programmes. Rev. Med. Vet. Entomol. 85: 355-368.

Pates, H. V., W. Takken, C. F. Curtis, P. W. Huisman, O. Akinpelu, and G. S. Gill. 2001. Host discrimination of Anopheles gambiae sensu stricto and An. quadriannulatus between a human and a calf. Trans. R. Soc. Trop. Med. Hyg. (in press).

Sharp, B. L., F. C. Quicke, and E. J. Jansen. 1984. Aspects of the behaviour of five anopheline species in the endemic malaria area of Natal. J. Entomol. Soc. S. Afr. 47: 251-258.

Snow, W.F. 1970. The effect of reduction in expired carbon dioxide on the attractiveness of human subjects to mosquitoes. Bull. Entomol. Res. 60: 43-48.

Takken, W., and D. L. Kline. 1989. Carbon dioxide and 1-octen-3-ol as mosquito attractants. J. Am. Mosq. Contr. Assoc. 5: 311-316.

Takken, W., and B.G.J. Knols. 1999. Odor-mediated behavior of Afrotropical malaria mosquitoes. Annu. Rev. Entomol. 44: 131-157.

Takken, W, W. Eling, J. Hooghof, T. Dekker, R. Hunt, and M. Coetzee. 1999. Susceptibility of Anopheles quadriannulatus Theobald (Diptera:Culicidae) to Plasmodium falciparum. Trans. R. Soc. Trop. Med. Hyg. 93: 578-580.

Trpis, M., and W. Hauserman. 1978. Genetics of house entering behaviour in East African populations of Aedes aegypti (L.) (Diptera: Culicidae) and its relevance to speciation. Bull. Entomol. Res. 68: 521-532.

Vale, G. A. 1979. Field responses of tsetse flies (Diptera: Glossinidae) to odours of men, lactic acid and carbon dioxide. Bull. Entomol. Res. 69: 459-467.

White, G. B. 1974. Anopheles gambiae complex and disease transmission in Africa. Trans. R. Soc. Trop. Med. Hyg. 68; $278-301$. 2001.
Received for publication 28 December 2000; accepted 6 July 